\title{
THE
}

2-1-1993

\section{Transverse Dynamics and Relaxation in Spin-Polarized or Two- Level Fermi Systems}

Alexander E. Meyerovich

University of Rhode Island, sfo101@uri.edu

K. A. Musaelian

University of Rhode Island

Follow this and additional works at: https://digitalcommons.uri.edu/phys_facpubs

Terms of Use

All rights reserved under copyright.

\section{Citation/Publisher Attribution}

Meyerovich, A. E., \& Musaelian, K. A. (1993). Transverse Dynamics and Relaxation in Spin-Polarized or Two-Level Fermi Systems. Phys.Rev. B, 47, 2897-2900. doi: 10.1103/PhysRevB.47.2897

Available at: http://dx.doi.org/10.1103/PhysRevB.47.2897

This Article is brought to you for free and open access by the Physics at DigitalCommons@URI. It has been accepted for inclusion in Physics Faculty Publications by an authorized administrator of DigitalCommons@URI. For more information, please contact digitalcommons-group@uri.edu. 


\title{
Transverse dynamics and relaxation in spin-polarized or two-level Fermi systems
}

\author{
A. E. Meyerovich and K. A. Musaelian \\ Department of Physics, University of Rhode Island, Kingston, Rhode Island 02881
}

(Received 31 August 1992)

\begin{abstract}
A microscopic theory is proposed for transverse dynamics and zero-temperature attenuation in polarized Fermi liquids. The transport equations are a set of two coupled equations in two "partial transverse densities," which do not reduce to a single equation in a mixed component of a single-particle distribution. The effective interaction is linked to an irreducible vertex by an integral equation, and cannot be given as a limit of a full vertex. A framework for a generalized nonlocal Landau theory is established. The spectrum of attenuating spin waves is calculated at arbitrary polarizations and densities.
\end{abstract}

The shortcomings of the Landau theory are, at present, some of the most widely discussed problems in condensed matter theory. Disturbingly, one of the oldest examples has remained unresolved for several decades: Landau theory fails to describe the transverse phenomena when the interlevel gaps are comparable with the Fermi energy. Furthermore, an adequate kinetic equation is still missing.

Transverse dynamics and relaxation are inherent features of any multilevel system. The transverse dynamics describes the behavior of off-diagonal (mixed) elements of a single-particle density matrix responsible for transitions between energy levels. If these levels correspond to spin states, then the transverse processes cover the motion of transverse components of magnetization. The transverse relaxation may be defined either as an attenuation of off-diagonal single-particle states, or as an attenuation of a collective mode reflecting coherent transitions of particles between energy levels (spin waves for spin systems). Such modes exist in all multilevel systems, at least in rudimentary form. ${ }^{1}$ If the transverse relaxation is large, the mode should be considered as a diffusive, rather than a coherent collective one. These descriptions of the transverse attenuation merge only for dilute systems.

The low-temperature transverse dynamics is important not only for traditional fields like spin dynamics and spin waves in Fermi liquids or spin-polarized quantum systems, ${ }^{1}$ itinerant magnetism, but also for less conventional systems like heavy fermions, marginal Fermi liquids, high- $T_{c}$ systems, and other objects with at least two-band carriers (see Ref. 2). General results presented below are applicable to many of these systems. For simplicity, we consider two-level particles (the pseudospin $\frac{1}{2}$ ), and use the terminology originating from magnetic systems. The generalization to other multilevel particles is straightfor- ward. The only constraint is that the bare energy gap between the levels for noninteracting particles should exhibit an insignificant momentum dependence. Such an assumption, being quite natural when dealing with spin states, is not always valid for levels of other nature like multiband solid-state quasiparticles. A momentum dependence of energy gaps will be considered elsewhere.

The problems of the Landau theory lie in an unclear nature of off-diagonal quasiparticles and temporal and spatial nonlocalities involving complex off-shell terms. The same factors are responsible for a zero-temperature transverse relaxation $\tau_{\perp}$ which does not increase as $1 / T^{2}$ like the longitudinal one remaining finite at $T \rightarrow 0 .^{3} \mathrm{Un}$ fortunately, an adequate transport equation for transverse phenomena is still absent encouraging the use of a Landau-like semiphenomenological equation ${ }^{4}$ for systems similar to highly spin-polarized Fermi liquids. Such a situation is very unsatisfactory.

Below we report the exact microscopic results for transverse dynamics which generalize and completely redefine the Landau theory. The general transport equation takes the form of a set of two coupled equations in some "partial transverse densities." The transverse relaxation is simply a dephasing between precessions of these densities in inhomogeneous systems resulting in an inhomogeneous broadening. We will also illustrate the general results in limits of low densities and polarizations. The detailed results will be published elsewhere.

At $T=0$ the Dyson equation for a small and slowly varying mixed spin component of the Green's function

$$
\begin{aligned}
\delta G_{\uparrow \downarrow}(P ; t, \mathbf{r})= & g(P)\left[G_{\downarrow \downarrow}(P+K / 2)-G_{\uparrow \uparrow}(P-K / 2)\right] \\
& \times \exp (-i \omega t+i \mathbf{k} \cdot \mathbf{r})
\end{aligned}
$$

reduces to

$$
\begin{aligned}
{\left[\delta \omega-\frac{\mathbf{p} \cdot \mathbf{k}}{m}-\Sigma_{\downarrow \downarrow}\left(P+\frac{K}{2}\right)+\Sigma_{\uparrow \uparrow}\left[P-\frac{K}{2}\right]\right) g(P) } & \\
& =-i \int \frac{d^{4} P^{\prime}}{(2 \pi)^{4}} \widetilde{\Gamma}_{\uparrow \downarrow, \downarrow \uparrow}\left(K ; P, P^{\prime}\right)\left[G_{\downarrow \downarrow}\left[P^{\prime}+\frac{K}{2}\right]-G_{\uparrow \uparrow}\left[P^{\prime}-\frac{K}{2}\right]\right] g\left(P^{\prime}\right),
\end{aligned}
$$


where $P=\left(p_{0}, \mathbf{p}\right)$ is the four-momentum, the energymomentum transfer $K=(\omega, \mathbf{k})$ is the cause of spatial and temporal nonlocalities, $\delta \omega=\omega-\Omega_{0}, \Omega_{0}$ is the bare gap between the levels (the Larmor frequency $2 \beta H$ for spin- $\frac{1}{2}$ particles), $\Sigma_{\uparrow \uparrow}, \Sigma_{\downarrow \downarrow}$, and $\widetilde{\Gamma}_{\uparrow \downarrow, \downarrow \uparrow}$ are the mass operators and the irreducible vertex in a particle-hole channel. The arrow indices mark the internal states (up and down spins).

In a homogeneous system $\mathbf{k}=\mathbf{0}$, the eigenvalue $\omega$ of Eq. (1) is equal to the bare gap $\Omega_{0}$ without any attenuation. The eigenvalue is quadratic in small inhomogeneities $\mathbf{k}, \omega=\Omega_{0}+\alpha k^{2}$. In cases when the densities are not too high and the interactions not too strong, the transverse relaxation $\tau_{\perp}^{-1}$ is proportional to $\operatorname{Im} \alpha:^{1}$

$$
\tau_{\perp}=\frac{2^{2 / 3} 9}{20} \frac{\pi^{2} \hbar^{4}}{a^{2} \operatorname{Im} \alpha} \frac{p_{\uparrow}^{5}-p_{\downarrow}^{5}}{\left(p_{\uparrow}^{3}-p_{\downarrow}^{3}\right)^{3}},
$$

where $p_{\uparrow, \downarrow}$ are the Fermi momenta for particles on upper and lower levels, and $a$ is the particles' scattering length. This expression can also be used for estimates at higher densities since $\tau_{\perp}$ is always related to $\operatorname{Im} \alpha$.

The integral equation (1) contains, as all similar expressions in the Fermi-liquid theory, a singularity associated with the poles of the Green's functions. Splitting the difference of two Green's functions into a singular and regular parts, $\phi_{s}$ and $\phi_{r}$, one can transform Eq. (1) to the equation with a regular kernel:

$$
\begin{gathered}
{\left[\delta \omega-\frac{\mathbf{p} \cdot \mathbf{k}}{m}-\Sigma_{\downarrow \downarrow}\left[P+\frac{K}{2}\right]+\Sigma_{\uparrow \uparrow}\left[P-\frac{K}{2}\right]\right] g(P)} \\
=\int \mathcal{C}\left(K ; P, P^{\prime}\right) \phi_{s}\left(P^{\prime} ; K\right) g\left(P^{\prime}\right) \frac{d^{4} P^{\prime}}{(2 \pi)^{4}}, \\
\mathcal{C}\left(K ; P, P^{\prime}\right)=\widetilde{\Gamma}_{\uparrow \downarrow, \downarrow \uparrow}\left(K ; P, P^{\prime}\right) \\
+\int \widetilde{\Gamma}_{\uparrow \downarrow, \downarrow \uparrow}(K ; P, Q) \phi_{r}(Q ; K) \mathcal{C}\left(K ; Q, P^{\prime}\right) \\
\times \frac{d^{4} Q}{(2 \pi)^{4}} .
\end{gathered}
$$

The function $\mathcal{C}\left(K ; P, P^{\prime}\right)$ serves as a generator of the transverse Fermi-liquid interaction. At zero polarizations the Landau function $\Gamma^{\omega}$ is defined by either of two equivalent relations:

$$
\begin{aligned}
\Gamma_{\uparrow \downarrow, \downarrow \uparrow}^{\omega}\left(\mathbf{p}_{F}, \mathbf{p}_{F}^{\prime}\right)= & \lim _{\omega \rightarrow 0 k \rightarrow 0} \lim _{\uparrow \downarrow} \Gamma_{\uparrow \downarrow \downarrow \uparrow}\left(\omega, \mathbf{k} ; 0, \mathbf{p}_{F} ; 0, \mathbf{p}_{F}^{\prime}\right), \\
\Gamma_{\uparrow \downarrow, \downarrow \uparrow}^{\omega}\left(P, P^{\prime}\right)= & \widetilde{\Gamma}_{\uparrow \downarrow, \downarrow \uparrow}\left(P, P^{\prime}\right) \\
& +\int \widetilde{\Gamma}_{\uparrow \downarrow, \downarrow \uparrow}(P, Q) \phi_{r}(Q) \Gamma_{\uparrow \downarrow, \downarrow \uparrow}^{\omega}\left(Q, P^{\prime}\right) \\
& \times \frac{d^{4} Q}{(2 \pi)^{4}} .
\end{aligned}
$$

At higher polarizations, when the poles of the Green's functions move away from each other, the Fermi-liquid interaction is defined exclusively by Eq. (2). The effective interaction resembles a nonlocal version of Eq. (3b), and cannot be related to a full vertex $\Gamma$ using any analog of Eq. (3a). This was one of the main obstacles which hindered previous attempts to derive a Landau-like theory for transverse processes (see, e.g., Ref. 4, and references therein).

Equation (2) is still a four-dimensional (4D) integral equation in the Green's function $g(P)$. We have to simplify it into a 3D transport equation, in some density $\delta n(\mathrm{p})$, eliminating the energy component $p_{0}$ of the fourvector $P=\left(p_{0}, \mathbf{p}\right)$. It turns out-and this is one of the main results - that Eq. (2) can be reduced only to a set of two coupled 3D equations in some partial transverse “densities" $\delta n_{\uparrow}$ and $\delta n_{\downarrow}$ :

$$
\begin{aligned}
W_{\Downarrow}(K ; \mathbf{p}) \delta n \|(\mathbf{p})=\frac{1}{2} \int & \frac{d^{3} p^{\prime}}{(2 \pi)^{3}}\left[\theta_{\downarrow}\left(\mathbf{p}^{\prime}\right)-\theta_{\uparrow}\left(\mathbf{p}^{\prime}\right)\right] \\
& \times \sum_{\|=\uparrow, \downarrow} F \| \rrbracket
\end{aligned}
$$

where the open arrow indices $\{\|\|$,$\} take the values \uparrow$ and $\downarrow$,

$$
\begin{aligned}
& F_{\Downarrow \|}\left(K ; \mathbf{p}, \mathbf{p}^{\prime}\right)=Z_{\Downarrow}\left(\mathbf{p} \pm \frac{\mathbf{k}}{2}\right] Z_{\Uparrow}\left(\mathbf{p}^{\prime} \pm \frac{\mathbf{k}}{2}\right) \Theta\left(K ; P_{\Downarrow}, P_{\Uparrow}^{\prime}\right), \\
& W_{\Downarrow}(K ; \mathbf{p}) \\
& =\left[\omega \mp \varepsilon\left\|\left(\mathbf{p} \pm \frac{\mathbf{k}}{2}\right) \pm \widetilde{\varepsilon}\right\| \neq \|\left(\mathbf{p} \pm \frac{\mathbf{k}}{2}\right)\right] Z_{\Downarrow}\left(\mathbf{p} \pm \frac{\mathbf{k}}{2}\right), \\
& \delta n \Downarrow(\mathbf{p})=g\left(P_{\Downarrow}\right), \\
& P_{\Downarrow}=\left[\varepsilon_{\Downarrow}\left(\mathbf{p} \pm \frac{\mathbf{k}}{2}\right]-\mu \mp \frac{\omega}{2}, \mathbf{p}\right), \\
& \theta_{\|}(\mathbf{p})=\theta\left[\varepsilon \Downarrow\left[\mathbf{p} \pm \frac{\mathbf{k}}{2}\right)-\mu\right] \\
& \varepsilon\left\|(\mathbf{p})=\frac{p^{2}}{2 m}+\Sigma \Downarrow\right\|(\varepsilon \Downarrow(\mathbf{p})-\mu, \mathbf{p}) \pm \beta H, \\
& \widetilde{\varepsilon}_{\Downarrow}(\mathbf{p})=\frac{p^{2}}{2 m}+\Sigma_{\Downarrow \Downarrow}(\varepsilon \rrbracket \neq \|(\mathbf{p} \mp \mathbf{k})-\mu \pm \omega, \mathbf{p}) \pm \beta H,
\end{aligned}
$$

the $Z$ functions are the residues of the Green's functions in their poles, and $(+)$ in $( \pm)$ corresponds to $\downarrow$. This doubling of the kinetic equation (4) is a result of the temporal nonlocality and is explained by an up-down asymmetry (resembling a particle-hole asymmetry) for dressed particles. It means that by turning down the spin of a spin-up particle and shifting its energy, one does not go into a spin-down state $\varepsilon_{\downarrow}(\mathbf{p})$, but into some different pseudostate $\widetilde{\varepsilon}_{\downarrow}(\mathbf{p})$. The partial densities $\delta n_{\uparrow}$ and $\delta n_{\downarrow}$ should be interpreted as two separate contributions to the off-diagonal distribution $\delta N_{\uparrow \downarrow}$ (the transverse magnetization) from slightly tilted spin-up and spin-down states $\delta n \|(\mathbf{p})$ $=\delta N_{\uparrow \downarrow}^{(\Downarrow)}(\mathbf{p})$.

The splitting of transverse density into two results in $2 \times 2$ matrices of transverse interaction operators $F\|\|$ and relaxation times $\tau_{\perp} \| \Uparrow$. These matrices become degenerate and the set (4) collapses into a single equation only at low polarizations or densities. Generally, all four components of $F\|\|$ and $\tau_{\perp}\|\|$ are different (the offdiagonal ones satisfy the Onsager relations).

Integral equations (4) constitute the analog of the Landau kinetic equation for transverse phenomena. An 
equivalent phenomenological theory should be formulated in the same way as Ref. 1 for longitudinal phenomena using an analogy between a polarized system and a binary mixture of spin-up and spin-down components. For longitudinal processes such an analogy is transparent. We do have two sorts of (quasi-)particles, $N_{\uparrow \uparrow}(\mathbf{p})$ and $N_{\downarrow \downarrow}(\mathbf{p})$, with different energy spectra, $\varepsilon_{\uparrow}(\mathbf{p})$ and $\varepsilon_{\downarrow}(\mathbf{p})$, and with separate equations of motion coupled through the Landau interaction and the collision operator. These (quasi-)particles remain near the Fermi surfaces throughout the interaction processes. Surprisingly, the same analogy holds for a description of transverse effects involving mixed states $N_{\uparrow \downarrow}(\mathbf{p})$. One usually assumes that there is only one type of off-diagonal state. This assumption would be valid when dealing with bare noninteracting particles without an up-down asymmetry. Then it is of no interest whether a spin-up or spin-down particle got flipped onto an $x y$ plane. This is not so for dressed particles. The particles are dressed differently depending on a spin projection, and the frequency and response depend on the type of the particles flipped even for the same density perturbations $\delta N_{\uparrow \downarrow}(\mathbf{p})\left[\widetilde{\varepsilon}_{\downarrow}(\mathbf{p}) \neq \varepsilon_{\downarrow}(\mathbf{p})\right]$. When the spins are untitled, we have two types of particles-spin up and spin down. If we tilt the magnetization, the molecular fields acting on tilted spin ups and spin downs are different at $\mathbf{k} \neq 0$ resulting in doubling of equations and dephasing (i.e., inhomogeneous broadening) of precession. The microscopic equations (4) involve not only the states $\varepsilon_{\uparrow}(\mathbf{p})$ and $\varepsilon_{\downarrow}(\mathbf{p})$, but also some pseudostates $\widetilde{\varepsilon}_{\uparrow}(\mathbf{p})$ and $\widetilde{\varepsilon}_{\downarrow}(\mathbf{p})$. These four types of states give us a four-component nonlocal generalized Landau operator $\hat{F}_{\|}\left(K ; \mathbf{p}, \mathbf{p}^{\prime}\right)(4)$ for transverse processes.

We solved Eq. (4) and found the expression for the ei- genvalue spectrum (spin waves) $\omega=\Omega_{0}+\alpha k^{2}$. The main obstacles to writing the solutions of (4) with $k^{2}$ accuracy, $\delta n \Downarrow=1+\mathbf{k} \cdot \mathbf{p} \delta n \|^{(1)}(\mathbf{p})$ via Fermi-liquid harmonics are $\theta$ functions in the kernel instead of $\delta$ functions as in an unpolarized case. One can calculate these integrals by a Newton-Cotes expansion with coefficients $a_{s}$, and write the solution in the operator form as

$$
\begin{aligned}
& \delta n_{\| s}^{(1)}=\sum_{l, \|}\left[\hat{W}_{\| s}^{(1)} \delta_{\|}\left\|-\delta p \hat{F}_{\|}^{(1)}\right\|_{s l} a_{l} p_{l}^{3} / 2 p_{s}\right]^{-1} \\
& \times\left[W_{\prod l}^{(k),(1)}+\frac{\delta p}{2} \sum_{\| n} F_{\|\|_{l n}^{(k),(1)}} a_{n} p_{n}^{2}\right. \\
& \left.+\frac{1}{2 p_{l}} \sum\left[F_{\| \Downarrow l \uparrow}^{(0)}+F_{\| \Downarrow l \downarrow}^{(0)}\right]\right], \\
& W_{\Downarrow s}=W_{\Downarrow}\left(\mathbf{p}_{s}\right), \\
& F_{\|\| s l}=F_{\| \Uparrow}\left(\mathbf{p}_{\mathrm{s}}, \mathbf{p}_{1}\right), \\
& F_{\Downarrow \| s \downarrow}=F_{\Downarrow \|}\left(\mathbf{p}_{s}, \mathbf{p}_{\downarrow}\right), \\
& K_{0}=(2 \beta H, 0) \text {, } \\
& W_{\Downarrow}^{(k)}(\mathbf{p}) \mathbf{p} \cdot \mathbf{k}=\frac{\partial W \Downarrow\left(K_{0} ; \mathbf{p}\right)}{\partial \mathbf{k}} \mathbf{k}, \\
& F_{\| \rrbracket}^{(k)}\left(\mathbf{p}, \mathbf{p}^{\prime}\right) \mathbf{p} \cdot \mathbf{k}=\frac{\partial F \| \rrbracket\left(K_{0} ; \mathbf{p}, \mathbf{p}^{\prime}\right)}{\partial \mathbf{k}} \mathbf{k},
\end{aligned}
$$

where the upper indices (0),(1) denote the angular harmonics, and $p_{s}$ changes with the index $s$ from $p_{\downarrow}$ to $p_{\uparrow}$ with the step $\delta p$. Finally, the eigenspectrum takes the form

$$
\begin{aligned}
& \alpha=\frac{Z_{\mathrm{eff}} \delta p}{N_{\uparrow}-N_{\downarrow}} \sum_{\|,\|, s} a_{s} p_{s}^{2}\left[\frac{1}{12}\left[W_{\Downarrow}^{(p),(2)}\left(p_{s}\right)-W^{(p),(0)}\left(p_{s}\right)\right] \delta n^{(1)}\left(p_{s}\right)+F_{\| \Uparrow}^{(k),(1)}\left(K_{0} ; p_{s}, p_{\uparrow}\right) p_{s}\right. \\
& +F_{\| \Uparrow}^{(1)}\left(K_{0} ; p_{s}, p_{\uparrow}\right)\left[\delta n_{\Downarrow}^{(1)}\left(p_{\uparrow}\right)+\delta n_{\|}^{(1)}\left(p_{\uparrow}\right)\right] p_{\uparrow}+F_{\Downarrow \|}^{(k),(1)}\left(K_{0} ; p_{s}, p_{\downarrow}\right) p_{s} \\
& +F_{\Downarrow \|}^{(1)}\left(K_{0} ; p_{s}, p_{\downarrow}\right)\left[\delta n_{\Downarrow}^{(1)}\left(p_{\downarrow}\right)+\delta n_{\|}^{(1)}\left(p_{\downarrow}\right)\right] p_{\downarrow} \\
& +\frac{\delta p}{8} \sum_{l} a_{l} p_{l}^{2}\left[\frac{2}{3} F_{\| \Uparrow}^{\left(k_{\alpha} k^{2}\right),(0)}\left(K_{0} ; p_{s}, p_{l}\right)-\frac{2}{3} F_{\| \Uparrow}^{\left(k_{\alpha} k_{\beta}\right),(2)}\left(K_{0} ; p_{s}, p_{l}\right)\right. \\
& \left.+F_{\Downarrow \|}^{\left(k_{\alpha} k^{2}\right),(1)}\left(K_{0} ; p_{s}, p_{l}\right)+F_{\| \Uparrow}^{(k),(1)}\left(K_{0} ; p_{s}, p_{l}\right)\left[\delta n_{\Downarrow}^{(1)}\left(p_{l}\right)+\delta n_{\|}^{(1)}\left(p_{l}\right)\right]\right), \\
& Z_{\mathrm{eff}}^{-1}=1-\frac{\delta p / 2}{N_{\uparrow}-N_{\downarrow}} \sum_{\|,\|, s} a_{s} p_{s}^{2}\left[\Sigma_{\| \Downarrow}^{\left(p_{0}\right),(0)}\left[P_{\| s}+\frac{K_{0}}{2}\right]+\delta p \sum_{l} a_{l} p_{l}^{2} F_{\| \Uparrow}^{\left(k_{0}\right),(0)}\left(K_{0} ; p_{s}, p_{l}\right)\right] .
\end{aligned}
$$

As a result, the real part of the spectrum and the zerotemperature attenuation are expressed via the zeroth, first, and the second angular harmonics of the functions $W$ and $F$ and their derivatives. The attenuation has as its sources (i) imaginary parts in single-particle energies $\varepsilon(\mathbf{p})$ and pseudoenergies $\widetilde{\varepsilon}(\mathbf{p})$ away from Fermi spheres; (ii) imaginary parts of energy components of the four-vectors $P_{\|}$in generalized Landau functions $F\|\|\left(K_{0} ; \mathbf{p}, \mathbf{p}^{\prime}\right)$ (4) and renormalization functions $Z \Downarrow(\mathbf{p})$; (iii) derivatives of vertex functions in off-shell directions which reflect spatial nonlocalities. Basically, for not very low polarizations and densities, the imaginary terms in $\alpha$ are of the same order as the real ones, and the (spin) waves are strongly damped. It is interesting to see how the spectrum behaves, and the imaginary terms disappear, in simple limiting cases. 
Low density Fermi liquids. Here we have a density expansion in $x=a N^{1 / 3}$ ( $a$ is the $s$-wave scattering length) which coincides with an interaction expansion. In the zeroth and first orders in $x$ the energies $\varepsilon\|(\mathbf{p})=\widetilde{\varepsilon}\|(\mathbf{p})$ are real, while the set (4) is degenerate and reduces to a standard gas equation ${ }^{1}$ with only one transverse relaxation time. The imaginary parts and the zero-temperature attenuation show up beginning with the second-order terms. However, for dilute gases, in the second order the set (4) still reduces to a single equation. The split between $\widetilde{\varepsilon}_{\|}(\mathbf{p}) \neq \varepsilon \|(\mathbf{p})$ (both with imaginary parts) and the doubling of kinetic equations with four characteristic times appear starting from the third-order density terms. These effects are missed in previous theories based on some form of a Landau or Boltzmann kinetic equation. ${ }^{4,5}$ The calculation in the main orders yields

$\alpha^{\prime}=\frac{5}{6 \pi a} \frac{E_{\uparrow}}{n_{\uparrow}} \frac{\left(1-d^{5}\right)}{\left(1-d^{3}\right)^{2}}-\frac{1}{3 m} \frac{1+d}{1-d}+\frac{9}{\pi^{3} m} \frac{F_{2}(d)}{\left(1-d^{3}\right)^{3}}$,

$\alpha^{\prime \prime}=\frac{4}{105 m} \frac{F_{1}(d)}{\left(1-d^{3}\right)^{3}}$,

where $d=p_{\downarrow} / p_{\uparrow}$; the functions $F_{1}(d)$ and $F_{2}(d)$ are given in Fig. 1 (cf. Ref. 5). These results assume a locality of the interaction. The nonlocality also leads to some zero-temperature attenuation but in a higher order in density than $\alpha^{\prime \prime}$ above. Such nonlocal attenuation is important if the range of interaction $r_{0}$ is larger than the scattering length (which may be the case for ${ }^{3} \mathrm{He}-{ }^{4} \mathrm{He}$ mixtures):

$$
\alpha_{n l}^{\prime \prime}=\frac{4 p_{\uparrow}^{2} r_{0}^{2}}{105 m} \frac{F_{3}(d)}{\left(1-d^{3}\right)^{3}} .
$$

Low fields and/or polarizations. Most of the interesting effects vanish in an approximation linear in the field. Then $\widetilde{\varepsilon}$ and $\varepsilon$ are real, system (4) is degenerate, and retains the classic Leggett-Silin form. The doubling of the equations, multiple transverse times, and the zerotemperature relaxation show up in the next order in $H$, when the corresponding term in $\alpha$ is field independent with an imaginary part-originating from all the terms in (4) - of the same order as the real one. The equations reduce to two subsets of three linear equations involving

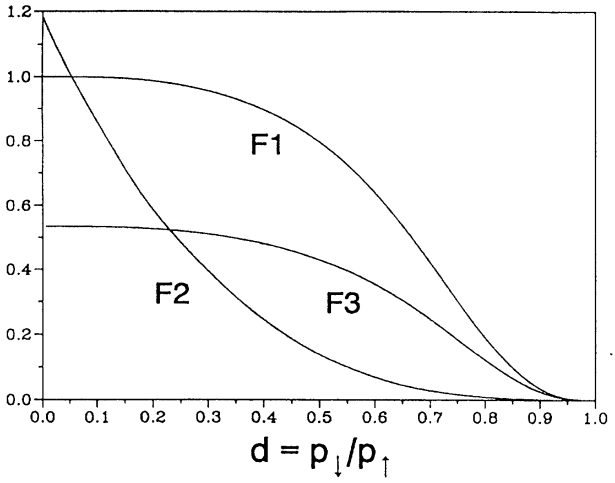

FIG. 1. Functions $F_{1}(d), F_{2}(d), F_{3}(d)$.

the values of the $\hat{F}$ matrices on three Fermi surfaces $p_{\uparrow}$, $p_{\downarrow}$, and $p_{0}$. This result for $\alpha$ has a simple structure, but is too cumbersome to be given here.

In summary, we have derived microscopic equations for the transverse dynamics and relaxation in polarized Fermi liquids at zero temperatures. The exact theory includes unavoidable spatial and temporal nonlocalities. The closed analytical expressions give the spin-wave spectrum with the zero-temperature relaxation at arbitrary polarizations. The main difference from phenomenological and semimicroscopic approaches is an unexpected doubling of transport equations as a result of temporal nonlocality. An analog of the Landau theory for transverse dynamics becomes rather peculiar with two sets of off-diagonal terms in spin particle and pseudoparticle states. The elements of the four-component nonlocal Fermi-liquid interaction operator for transverse processes are expressed via an off-diagonal element of the irreducible vertex, and do not reduce (as for unpolarized systems) to any limit of a full vertex. At low polarization or low density, the theory reproduces the well-known expressions though in a nontrivial way. The results are applicable to transverse dynamics in any Fermi liquid of two-level particles. The detailed results will be given elsewhere.

The work was supported by NSF DMR-9100197.
${ }^{1}$ A. E. Meyerovich, in Helium Three, edited by W. P. Halperin and L. P. Pitaevski (North-Holland, Amsterdam, 1990), pp. 760-869.

${ }^{2}$ C. M. Varma et al., Phys. Rev. Lett. 63, 1996 (1989); P. W. Anderson, ibid. 67, 2092 (1991).
${ }^{3}$ A. E. Meyerovich, Phys. Lett. 107A, 177 (1985).

${ }^{4}$ C. R. Sanchez-Castro, K. S. Bedell, and S. A. J. Wiegers, Phys. Rev. B 40, 437 (1989); K. F. Quader and K. S. Bedell, J. Low Temp. Phys. 58, 89 (1985).

${ }^{5}$ J. W. Jeon and W. J. Mullin, Phys. Rev. Lett. 62, 2691 (1989). 